\title{
Gradhiva
}

\section{Guillaume Lachenal, Le Médecin qui voulut être roi : sur les traces d'une utopie coloniale}

Paris, Éditions du Seuil, coll. « L'univers historique », 2017

\section{Florent Papin}

\section{(2) OpenEdition}

\section{Journals}

Édition électronique

URL : http://journals.openedition.org/gradhiva/3588

DOI : 10.4000/gradhiva.3588

ISSN : 1760-849X

\section{Éditeur}

Musée du quai Branly Jacques Chirac

Édition imprimée

Date de publication : 23 mai 2018

Pagination : 262-264

ISBN : 978-2-35744-110-1

ISSN : 0764-8928

\section{Référence électronique}

Florent Papin, «Guillaume Lachenal, Le Médecin qui voulut être roi : sur les traces d'une utopie coloniale», Gradhiva [En ligne], 27 | 2018, mis en ligne le 23 mai 2018, consulté le 07 janvier 2021. URL : http:// journals.openedition.org/gradhiva/3588; DOI : https://doi.org/10.4000/gradhiva.3588

Ce document a été généré automatiquement le 7 janvier 2021.

(c) musée du quai Branly 


\section{Guillaume Lachenal, Le Médecin qui voulut être roi : sur les traces d'une utopie coloniale}

Paris, Éditions du Seuil, coll. « L'univers historique », 2017

Florent Papin

\section{RÉFÉRENCE}

Guillaume Lachenal, Le Médecin qui voulut être roi : sur les traces d'une utopie coloniale, Paris, Éditions du Seuil, coll. «L'univers historique », 2017, 353 p. 


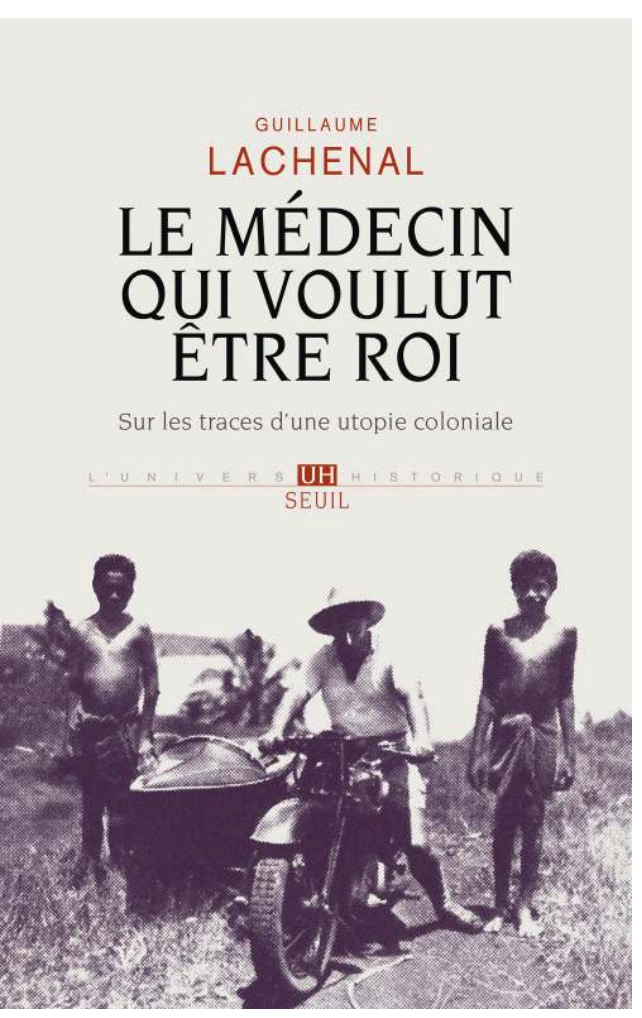

1 «Tout est vert, rouge et calme » : la formule revient à deux reprises sous la plume de Guillaume Lachenal. À une centaine de pages d'intervalle (p. 14, p. 169), à des milliers de kilomètres de distance, des contrées se mêlent par la grâce de la poésie, des époques se croisent. Un complexe scolaire camerounais d'Abong-Mbang paraît épouser la superficie de l'île de Wallis. Du Cameroun à la Polynésie, il y a comme un fondu enchaîné dans la vision de ces "vérandas qui se ressemblent ", une correspondance entre « les autochtones et les climats, les amours et les accents, les fruits du jardin, les couleurs de la terre, [...] les saisons des pluies ». L'auteur, d'ailleurs, laisse le glissement s'opérer : « Je passe mon temps à penser au Cameroun. » (p. 177)

Répondant aux attentes actuelles de la discipline, Guillaume Lachenal donne des couleurs à l'écriture de l'histoire. Il lui confère texture et même tessiture - au point d'imaginer que les faits qu'il relate puissent l'être « en fredonnant » (p. 120).

L'ouvrage s'attache à la figure et aux réalisations d'un médecin du Corps de santé colonial, Jean Joseph David, au temps où celui-ci se vit attribuer des pouvoirs disciplinaires. À Wallis tout d'abord, dans les années 1930, puis dans le Haut-Nyong, au Cameroun, durant la Seconde Guerre mondiale. Dans ces territoires sous contrôle, David entreprit de gouverner les hommes, les terres et les mœurs, s'essayant à un dirigisme vitaliste total. Il fit pour cela construire des hôpitaux, tracer des routes, défricher les sols, modifier les plantations. Il mit en place des outils statistiques, monta des équipes de football, apprit les idiomes. Il contraignit la force de travail, déplaça des hommes, emprisonna, fit marquer à la chaux d'une croix blanche sur le crâne les malades de la trypanosomiase. Il frappa et causa la mort prématurément, par accident, par épuisement.

4 L'auteur fouille de son écriture l'espace façonné par un homme de pouvoir, aussi trouble qu'ont pu l'être le moment et l'environnement. Et il le fait sans coquetterie, 
mais en suivant au contraire un protocole méthodologique fort, assumé, où le travail du texte devient le lieu du cheminement historique. L'ouvrage qu'Ivan Jablonka a consacré à la question des rapports entre l'écriture de l'histoire et de la littérature ${ }^{1}$ est à la fois trop ample et suffisamment clair pour qu'il y ait lieu d'en rappeler ici les apports : disons simplement que Le Médecin qui voulut être roi aurait pu prendre pour sous-titre L'histoire est une littérature contemporaine.

5 La production d'un «texte-recherche", pour reprendre la notion d'Ivan Jablonka, participe assurément d'un plaisir d'écriture. Celui-ci est manifeste chez Guillaume Lachenal, dont le style fluide et la dextérité narrative sont notables, suscitant en retour un grand plaisir de lecture. Mais la forme ne répond pas seulement d'elle-même. Elle épouse dans le cas présent une démarche de recherche, annoncée dans l'incipit - lequel revendique au passage la part de littérarité de l'entreprise : Le Médecin qui voulut être roi est une enquête sur des traces.

6 Les traces laissées par le personnage sont plurielles, comme l'a été son action visant à éradiquer les maladies endémiques - du sommeil en premier lieu -, à redresser la démographie, à régénérer les forces productives et potentiellement combattantes. La gratitude envers la France et son œuvre suivrait. Ce que l'époque commençait déjà à nommer une politique de développement ${ }^{2}$.

7 Les traces, l'archive écrite en contient, tapies dans un rapport à la hiérarchie, une note de mission, un décret de nomination, un article. Il y a également la correspondance de David, bien mince, les missives de missionnaires l'ayant côtoyé à Wallis. Il se signale aussi par ses réalisations, en dur, dont il reste quelques vestiges, comme la maternité d'Abong-Mbang ou la léproserie de Kwoamb, dans le Haut-Nyong. Il a enfin laissé son empreinte là où il a séjourné : on retrouve son souvenir dans des récits locaux, des prénoms, des visions, des parfums.

8 Les traces du personnage sont plurielles, mais demeurent éparses, dérobées, incertaines. Guillaume Lachenal n'a pas son pareil pour les révéler. Certes, est trace, indice ou source ce que le chercheur définit comme tel. Ivan Jablonka ne dit-il pas à propos des faits que l'historien les « "invente" » dans la mesure où il « les cherche, les établit, les sélectionne, les ordonne, les hiérarchise, les relie en chaîne explicative » ? Et s'il est illusoire de croire que « la matière est donnée à l'avance et qu'il suffit d'aller la recueillir dans le réel » (p. 248), il le serait tout autant d'imaginer qu'elle se fabrique de manière arbitraire.

9 L'intérêt majeur et la qualité première du Médecin qui voulut être roi sont de chercher à élaborer les faits à travers une poétique. Les traces de David affleurent à proportion de l'habileté de l'historien à nouer un rapport sensible avec les lieux qu'il traverse, les atmosphères qu'il perçoit, les souvenirs qu'il convoque chez ses interlocuteurs : ce que Lachenal nomme, à la suite de Laurent Olivier, une connaissance "relationnelle » du passé (p. 207). Dans le fouillis des mémoires, dans le silence des lieux, sens et perceptions forment le négatif sur lequel s'impriment les marques de ce "passé [qui] “travaille " le présent, le hante» (p. 205).

On doit à cette poétique plusieurs séquences admirables, telle cette déambulation de l'auteur aux abords de l'hypnoserie de Madouma, où les arbres plantés dans les années 1940 pour éloigner les mouches tsé-tsé, "venus du passé ", sont par leur pestilence «la trace de la maladie du sommeil » (p.74); ou encore, de l'autre côté du globe, le regard posé sur les bas-côtés des routes de Wallis, dont la taille impeccable est attribuée aux injonctions de David, exigeant à l'époque que chaque famille prenne en 
charge l'entretien du réseau de voirie. «Le "temps de David ", écrit-il, c'est l'odeur de l'essence et le bruit de la tondeuse" (p. 208). On l'aura compris: la poétique de Guillaume Lachenal est une épistémologie. Elle participe d'une conception de l'histoire qui fait « de la rencontre inattendue avec la trace le point de départ de l'enquête, et non un "chapitre d'ouverture" ou un épilogue du vrai livre d'histoire» (p. 205). L'historien s'inscrit en cela dans le sillage d'une Nancy Rose Hunt et de son historiographie du « débris ${ }^{3}$ ».

11 En faisant du «détour par les traces » le ressort de son enquête, l'historien entend arriver à « une manière de poser différemment la question du passé et du présent, de penser leur rapport d'imbrication et d'engendrement mutuel» (p. 205). De là à considérer que la méthodologie de recherche et l'écriture deviennent le fin mot de l'histoire, ou pour le dire avec la finesse d'Ivan Jablonka, que «le cœur du livre ne serait plus le récit historique, mais le récit du raisonnement historique» (p. 296), il n'y a qu'un pas, que le lecteur est enclin à franchir, encouragé par l'auteur lui-même lorsqu'il affirme que "les archives sont " sujets" autant que "sources" " (p. 300). Une position de plus en plus pressante à mesure qu'on avance dans la lecture, et qui suscite un certain désarroi - mêlé d'enthousiasme pour plusieurs développements remarquables de l'historien, telles son anthropologie des pouvoirs à Wallis ou sa lecture de la mémoire de David à travers les mythes uvéens.

12 En toute logique, en toute attente devrait-on écrire, la conduite d'une enquête appelle une élucidation ou, à défaut, un cheminement en direction de la vérité. Il en va ainsi de la justice, de l'histoire, comme de tout exercice probatoire. Ce qui n'exclut pas les détours, précisément. Ni les retours en arrière. Ni les impasses. Dans ce registre aussi, d'ailleurs, Guillaume Lachenal excelle, livrant un modèle de "contre-épistémologie " (Jablonka 2014: 180) par l'expression récurrente de ses questionnements, de son imaginaire, de ses précautions. Au point, parfois, de flirter avec le maniérisme, à l'image de cet irritant : «Le colonel David est-il " devenu cinglé ", comme le suggèrent les missionnaires? Je ne pense pas, en tout cas je ne sais pas » (p. 147).

13 Ce dont le lecteur est sûr, en revanche, c'est qu'au terme de l'ouvrage, il ne connaît pas David, du moins pas véritablement. Parfois en vient-il même à se demander s'il l'a réellement approché, dans le plein de ses intentions, dans la nature de ses ambitions, dans le calcul de ses actes. Sans doute est-ce beaucoup demander au chercheur, au poète, au romancier, au juge ou encore au psychologue, bref, à quiconque essaie de comprendre ce que les hommes font. Toujours est-il que la part d'insaisissable de la figure de David interroge le geste de l'historien. Ce qui est d'autant plus déroutant qu'il ne s'agit pas là d'une défaillance méthodologique, tant s'en faut. On le redit: Guillaume Lachenal n'a pas son pareil pour créer des sources, faire parler les lieux, interroger les silences et le passé qui ourdit le présent. Le portrait aux facettes surabondantes livré par l'enquête finit même par étourdir, campant un personnage kaléidoscopique qui n'offre aucune image fixe.

Osons une hypothèse: le livre se referme sans que le lecteur ne connaisse véritablement David parce que l'auteur lui-même n'a pas souhaité véritablement le connaître. Peut-être la chose était-elle annoncée dès le début du livre, et peut-être convenait-il de prendre au pied de la lettre l'idée d'une enquête "sur » les traces : une enquête qui prenne la trace pour objet et destination, et non une enquête conduite en suivant des traces, additionnant les indices comme autant de signaux directionnels. On concevra d'ailleurs que la poétique de la démarche ait encouragé cette histoire en 
pointillé, tenue par les vides, appelant précisément un traitement pointilliste du personnage, où la distance vis-à-vis du motif ferait pendant au flou de la photographie de couverture, sur laquelle le visage de David n'est qu'une tache sombre d'où rien n'émerge, sinon le cylindre d'une cigarette.

Il n'est pas illégitime de postuler ici le volontaire brouillage biographique, tant l'historien parait craindre les limites inhérentes aux récits de vie. L'illusion biographique est l'un de ces écueils qui conduisent l'auteur à revendiquer la possibilité d'une «abiographie» (p. 250). Mais c'est la tentation métonymique que Guillaume Lachenal semble redouter par-dessus tout, entendant par exemple faire de son enquête à Wallis "une étude de cas qui ne serait l'instance d'aucune généralité »(p.179), a fortiori s'agissant d'un personnage dont la trajectoire traverse des catégories vastes et englobantes : colonialisme, impérialisme, biopolitique, médecine sociale.

Seulement, en voulant décharger David d'une histoire qui serait plus grande que lui, dont celui-ci ne serait en quelque sorte qu'un acteur en plus et en moins, « au second degré » (p. 282), contemplant sa position comme un "quiproquo» (p. 302) prêtant éventuellement à sourire ( il est impossible de savoir s'il souriait de ses propres excès ", p. 283), Guillaume Lachenal a l'air de considérer comme étant hors sujet ce qui pourrait être retenu à charge contre le médecin-commandant. Comme s'il préférait ne pas juger David en historien au motif que la vérité du personnage se situe ailleurs, au fond sur un plan ontologique : celle d'un homme aux prises avec la difficulté d'être, dont il s'accommode à sa façon.

On comprendrait dès lors mieux le souhait de Lachenal de ne pas faire un « récit d'hubris et de némésis », préférant lire cette histoire « comme un conte moral » (p. 301), tout comme son application à ajouter sans cesse à la complexité du personnage, à la fois «tranquille et violent, habile et maladroit, aimant et haineux, aimé et haï » (p. 250). Une complexité que l'historien tend à considérer avec indulgence, voire complaisance, quand il présente David comme un "incompris » (p. 291), un «meneur d'hommes » (p. 288) certes autoritaire, mais qu' « il ne s'agit pas d'accabler», qui «fit ce qu'il put avec l'énergie et le courage qu'on lui connaît » (p. 270). Enfin, on comprendrait mieux sa lecture surprenante, et à vrai dire peu convaincante, des ambitions de David et de ses pairs (Jamot, Koch) comme étant la marque d'un habitus médical de l'échec, doublé "d'une mégalomanie [qui] est une maladie professionnelle des médecins » (p. 301).

La richesse de l'ouvrage suggère pourtant qu'au milieu des traces de David, dans le blanc des pointillés, gisent des vérités pleines, fragmentaires certes, mais suffisamment signifiantes pour comprendre ce que l'homme a fait, en dégager une possibilité de jugement et une politique de l'histoire. Or c'est peut-être bien ce dont souffre l'enquête : l'absence d'une politique, de la « colère de la vérité » chère à Ivan Jablonka (2014 : 158). Il a peut-être manqué aussi la colère du poète face à des mots piégeux en diable - modernité, utopie, développement, progrès, nostalgie... -, dont la surdétermination fait discours par-delà la langue de l'historien. Aussi la poétique de la trace attendait-elle peut-être son pendant sémantique, une fragmentation du verbe (et pas seulement de la narration) qui aurait été la condition d'une intelligence renforcée des faits. Cette politique de la poétique aurait pu donner une direction plus franche à la quête du David historique, au vrai de ses expériences, celle du Haut-Nyong notamment, dont l'auteur finit par juger qu'« elle n'a pas eu lieu » (p. 133).

19 À moins... à moins que dans cette affirmation, dans la charge tragique qu'elle recèle autant que dans sa part d'artifice, on n'atteigne le vrai de l'ouvrage: une tentative 
audacieuse de pousser au plus loin la possibilité d'écrire en historien une histoire qui n'a pas eu lieu; expérimenter la possibilité de rêver l'histoire dans un régime d'historicité. Et ce n'est pas tant l'inclination de l'auteur à superposer la figure du général Patton à celle de David qui le laisse imaginer, ni l'allusion répétée à James Brooke, le rajah blanc dont s'est inspiré Kipling pour L'Homme qui voulut être roi. Non, le rêve d'histoire de Guillaume Lachenal surgit bien plutôt à un moment précis, acmé de la poétique de l'entreprise dans ce qu'elle a de plus puissant, fructueux et inachevé : voici le chercheur à l'aéroport, arrivant de Wallis en correspondance à Nouméa. À ses côtés se tient " une dame âgée, mince, belle, avec de grands cheveux blancs : Thérèse Brial [...], l'ex-belle-sœur de David». De son propre aveu, l'historien n'a «jamais été aussi proche du roi David». Mais craignant d'importuner la dame, redoutant plus sûrement de dissiper le rêve, il n'ira pas à sa rencontre. Les bagages sont sur le point d'arriver, le vol pour Paris s'annonce. « Le passé restera à sa place. » (p. 286)

\section{NOTES}

1. Ivan Jablonka, L'histoire est une littérature contemporaine : manifeste pour les sciences sociales, Paris, Éditions du Seuil, coll. « La librairie du Xxi ${ }^{\mathrm{e}}$ siècle ", 2014, 339 p.

2. Lire à ce propos l'article de Véronique Dimier, «Politiques indigènes en France et en GrandeBretagne dans les années 1930: aux origines coloniales des politiques de développement", Politiques et Sociétés 24 (1), $2005: 73$ - 99.

3. Nancy Rose Hunt définit sa méthode comme «visant à traquer les couches archéologiques d'objets, de pratiques et de mots [...], privilégiant de plus en plus le concept de " débris " colonial [...] comme concept et méthode dans l'histoire ethnographique [...] : ces objets sont saturés de mémoire et ils constituent des condensés d'histoire". Voir «Espace, temporalité et rêverie : écrire l'histoire des futurs au Congo belge », Politique africaine 135, 2014 : 115-136.

\section{AUTEURS}

\section{FLORENT PAPIN}

Florent.PAPIN[at]quaibranly.fr 